\title{
Review on Construction Procedures of Driving Cycles
}

\author{
L. CZÉGÉ1 , A. VÁmosI² I. KoCSIS ${ }^{3}$ \\ ${ }^{1}$ University of Debrecen, Faculty of Engineering, Department of Mechanical Engineering, \\ czege.levente@eng.unideb.hu \\ 2University of Debrecen, Faculty of Engineering, Department of Basic Technical Studies, \\ vamosi.attila@eng.unideb.hu \\ ${ }^{3}$ University of Debrecen, Faculty of Engineering, Department of Basic Technical Studies, \\ kocsisi@eng.unideb.hu
}

Abstract: The goal of this paper is to give an overview of the literature of construction techniques of driving cycles. Our motivation for the overview is the future goal of constructing our own driving cycles for various types of vehicles and routes. This activity is part of a larger project focusing on determination of fuel and energy consumption by dynamic simulation of vehicles. Accordingly, the papers dealing with sample route determination, data collection and processing, driving cycle construction procedures, statistical evaluation of data are in our focus.

\section{Introduction}

The possibilities of construction of driving cycles are discussed in several technical papers, and many different processes have been developed over the past decades. These methods are fundamentally similar in working with large amounts of long-term data collected under real-world conditions and applying some statistical procedure to develop the representative driving cycle of the desired length. However, based on the review of the literature, it can also be concluded that there may be significant differences in the techniques and tools used to collect data, as well as in the data processing and in the applied statistical methods, resulting in different driving cycles. The validity of the various different techniques cannot be the subject of debate, but the question arises, which procedure should be used in a certain case. This depends on for example the purpose for which the driving cycle is being used, the quality and quantity of data, and the possibilities of collecting data.

The goal of this paper is to give an overview of the relevant literature. Our motivation for the overview is the future goal of constructing our own driving cycles for various types of vehicles and routes. The need to develop representative driving cycles is related to a larger project focusing on determination of fuel and energy consumption by dynamic simulation of vehicles. Accordingly, the papers dealing with sample route determination, data collection and processing, driving cycle construction procedures, statistical evaluation of data are in the focus of this literature research. 


\section{Application of driving cycles}

In practice, to determine the emission and energy consumption of vehicles, it is a common method to subject the vehicles to test procedures whereby the use of the vehicle - in relation to consumption - is similar to that experienced in real-world traffic conditions [13]. The artificial load simulating the real loads shall be such that it is statistically representative from point of view of the consumption and emission, as well as the variables describing them [6]. In practice, the load simulating the real loads is given in the form of so-called driving cycles [30]. The driving cycle is primarily a function of the route, which describes the change in vehicle speed over time. Some studies [28] have highlighted that the cycle can depend on other factors besides the route, such as vehicle type, energy source, driver. Of course, depending on the actual situation to be simulated, different driving cycles can reproduce the actual loading in a representative way. There are generally recognized driving cycles such as: WLTC (Worldwide Harmonized Light Duty Vehicles Test Cycles), which are primarily intended to measure and compare vehicle consumption and emissions under the same test conditions. These driving cycles have been designed to be representative of the global average vehicle load [29].

Many studies have shown that these general driving cycles, due to their global nature, are not accurate enough to adequately estimate emissions and energy use for specific geographic areas, cities or special purpose vehicles (e.g. public transport, freight transport, utility vehicles) [4] [12] [25] [26]. It is generally accepted in the technical literature that accurate determination of this data requires driving cycles that are more representative of the typical use of a given region, city, or mode of use [11]. Accordingly, many different driving cycles have been published in recent years, all of which are indeed very different from the global driving cycles.

\subsection{Driving cycles representative for certain geographical areas}

Some of the technical papers dealing with driving cycles aim to develop a cycle specific to a geographical area or a city. The [2] introduces a driving cycle for the morning peak driving patterns in Sydney. The paper introduces a detailed description of the technique of data collection as well as the method of the analysis. The Sydney Driving Cycle was assembled using 8 segments with a total duration of $10 \mathrm{~m} 37 \mathrm{~s}$. The [3] proposes a driving cycle derived from driving behaviour and real traffic conditions in Mexico City. To develop the driving cycle the authors employed the chase-car approach, and evaluated a total of 108 trips $(1044 \mathrm{~km})$, and $60 \mathrm{~h}$. The new cycle lasts 1360 seconds with a distance of $8.8 \mathrm{~km}$, and average speed of $23.4 \mathrm{~km} / \mathrm{h}$. The paper [5] gives a detailed description of the LA92 driving cycle, which was developed to treat concerns related to the FTP's lack of high speed and high acceleration activities. The paper reveals that even this cycle has problems in reflecting the true driving patterns due to the "quasi-random" approach for microtrip selection. The authors constructed a new driving cycle (LA01) based on a new stochastic method. The [7] deals with the driving characteristics in a developing city of China. The authors used the average speed, average running speed, average acceleration rate, average deceleration rate, mean length of a driving period, time proportions of driving modes, average number of acceleration-deceleration changes, root mean square acceleration, and positive acceleration kinetic energy as parameters to characterise the driving 
patterns. In [11], to obtain a driving cycle for Singapore, the authors apply a novel method based on the GPS data. Through the matching of GPS coordinates with the timestamps of collected data on map road types the time periods are clearly identified without the need of estimation via cluster analysis. The paper [16] deals with the construction of a driving cycle for Hong-Kong for the assessment of fuel consumption and exhaust emission models. To develop the driving cycle the authors selected 20 short driving periods so, that the values of the nine assessment parameters can best fit those of the whole collected data. To determine the representativeness of the cycle the concept of coefficient of variation is applied. The final driving cycle is chosen from ten different cycles developed by random selection approach. The paper [17] introduces three driving cycles (urban, sub-urban and highway driving) for Hong Kong. The authors applied the common random selection process, with two refinements: a more reasonable cycle length calculation method and a more stringent criteria for the selection of the best driving cycle. The most representative cycle from the 10 developed candidate cycles were selected based on the performance value and the speed acceleration probability distribution. The [20] discusses the development of a driving cycle in the area of Tamilnadu, India. The authors selected a major road of $7 \mathrm{~km}$, and collected the data for a period of 30 days. For data collection they applied the on-board method using a data acquisition control system, composed of an infrared optical sensor that is pointing towards the vehicle wheel axle. The [27] deals with the driving cycle construction for the urban area for the city of Edinburgh. The authors applied the car chase technique to collect real-world traffic data with an instrumented car. They developed a new statistical method called TRAFIX to analyse the collected data. For developing the driving cycle a weighting factor based on the traffic flow of the certain roads were involved.

\subsection{Driving cycles representative for special purpose vehicles}

Depending on the final goal, special driving cycles are needed, which represent a special type of vehicles or a special application. There are several examples when buses are in focus [15]. The [8] gives a comparison of the Markov-chains method, the Micro-trips method and the deterministic Minimum weighted difference - characteristics parameters method for a certain bus route. In the study five driving cycles are developed using the methods. The basis of comparison is the representativeness of the driving cycle, which can be calculated using the Characteristic parameters such as mean speed, positive kinetic energy, etc., as well as the speed acceleration probability distribution. The same group of authors compared the mentioned methods with the Fuel-based method as well in [9]. During the data collection the ECU of the buses, GPS system, altimetry measurements and emission measurement were applied collecting fuel consumption, position as a function of time, and CO, CO2, NO and NO2 values at $1 \mathrm{~Hz}$. Besides developing various driving cycles, the study introduces a methodology to assess the representativeness of driving cycles. The [18] combines the K-means cluster method with principal component approach to develop a representative driving cycle for urban buses in a Chinese city Fuzhou. The micro-trips are described by 15 characteristic parameters, which were then reduced through the Principal component analysis to reduce the complexity of the calculations. A novel approach is to apply the Silhouette value to evaluate the appropriateness of clustering. The micro trips used for the construction of the candidate driving cycle were selected by the size of the cluster center. Finally, a target driving cycle was achieved based on 6 characteristic parameters (percentage of 
accelerating, percentage of idling, percentage of cruising, mean velocity, mean acceleration, and the standard deviation of acceleration). The [19] compares a common and a novel construction method of driving cycles for a bus with a fixed route in Shanghai. In case of the common method, instead of the widely used micro-trip approach, it applies kinematic segments, which refers to the driving cycle segments with fixed time-steps. In the second, novel method a new segment split method based on bus stopping is applied. According to the comparison the improved, bus-station-based method is more representative. The paper [15] presents the development of a driving cycle for electric bus traveling a certain route in Hong-Kong. The authors used an IPhone 6s and a GPS application to collect the location and speed information of the bus. After determining the micro-trips and building the candidate cycle by random selection, they used 13 parameters for assessment. In addition, they applied the Average absolute percentage error and the Vehicle Speed Distribution as well.

In several cases the driving cycles are made for electric vehicles. It is assumed that the driving cycles of electric vehicles are significantly different from those of internal combustion engine vehicle, due to the differences in the torque, power, and braking characteristics [10]. In the paper [1] the driving cycle of electric special-purpose vehicle is investigated. The authors introduce the methods of principal component analysis, clustering analysis, random assortment and correlation coefficient verification methods which were applied to develop the cycle. For data collection a GPRS communication system were used. The [22] deals with the development of driving cycles for Shenyan, China, with the goal of determination of the control strategy of electric vehicles. The authors applied the principal component analysis to reduce the dimensionality of the collected data by finding a new set of variables. They used k-means clustering to group the kinematic fragments. In the [23] the authors present three methods to develop the driving cycle of Beijing based on the data of 40 electric taxis. These are the Markov Monte Carlo method, micro-trip (Random) method and the micro-trip (Sequence) method. The data is taken from the National monitoring and Management Platform for electric vehicles which collects the realtime data of 200 thousand electric vehicle in Beijing.

One can find unique driving cycles as well, as for example the one for tricycles in Metro Manila, Philippines [21]. The authors instrumented a common tricycle with photodiode sensor to measure the rpm of the wheel, and gathered data following the chase car method. The data then were analysed and a driving cycle were developed based on the Markov chain method. The candidate cycle with the best velocity-load fit with statistically similar parameters was selected to form the drive cycle. A driving cycle for motorcycles are introduced in the [12] for Hanoi, the capital of Vietnam. The authors selected 10 routes in the city to make the analysis, a second-by-second vehicle speed data were collected by GPS and speed vector sensor equipment, then micro-trips bounded by idling times were identified, then by random selection the candidate cycle was established, for which the representativeness were checked by the 12 parameters as a set of assessment criteria. The [14] presents two driving cycles for special purpose utility vehicles. A not common technique was used for data collection: a data logger was connected to the vehicle's OBD port to record the speed, to ensure to capture true driver behaviour, the drivers were not instructed, nor informed about the data collection. 


\section{Hardware and software requirements}

\subsection{Hardware for data collection}

To create driving cycle we need traffic information from vehicles such as velocity, distance travelled, geographic position, fuel and energy consumption, etc. These are requiring data collection. The data may derive from test vehicles, which shall be equipped with a measuring system [31] [32] [33] [34], or come from different company vehicles that already have on-board measurement and data collection systems, such as buses of transport companies [35] [36] [37], cars of taxi companies [38], or trucks of logistic companies [39] [40], but we can also use values which are measured by population of a city or a country [41] or collect from pre-built databases [42].

For installed measuring systems, we can connect to the vehicle's On-Board Diagnostic (OBD) computer with Controller Area Network (CAN bus) communication, and read data, usually velocity and distance travelled, but also collect other information such as engine performance and fuel consumption [31] [34] [35] [39] [43]. If the vehicle cannot be fitted with a device, for example on a motorcycle, because of the size of the instrument is too large, then a mobile device can be used to measure [33], for example, a handheld GPS receiver [31] [35] or if we use multiple vehicles, and it is not necessary to make more measurement in the same time, we can also move the device [41].

A global positioning system (GPS) device can also be used as a standalone measurement tool [38] [41] [44], since drive cycle can be created based on geographic location data (latitude, longitude, altitude) and velocity measurement or calculation. However, the reception of GPS signals is rather inaccurate due to external environmental influences (such as reflections from high-rise buildings in downtown), therefore measured values should be filtered, corrected and refined [45] [46]. That is why GPS is usually used as a supplement (taking into account altitude values, identifying stopping points, etc.) [35] [47] [48] or checking and correcting the values measured with on board instrument [32] [39] [43]. They can also be used to determine the nature of travel (downtown, suburban, express) or to connect the result of measuring to Geographic Information System (GIS) software [42] [43] [49].

Data acquisition tools for measurement are also available on the market in complex form [35] [39] or as applications for mobile phones and tablets [47]. Storage of data may be large depending on the number of measured values, so in some cases the data is not stored locally, but is transmitted wirelessly via GSM or GPRS to a central server [43] [50]. However, these systems may experience loss of data due to an error or intermittent communication, which can be eliminated by checking and preprocessing the data, but it requires additional work and time.

We can also create our own data acquisition device with microcontroller control, such as the tool described in [51]. The advantage of ready-to-use devices is that they do not need to be upgraded, they are immediately usable, but in many cases the data available is limited. The benefit of building our own measuring system is that, it can be refined to suit the data what we need. It can be supplemented with other sensors such as fuel consumption meter [37], emissions meter [52] or battery charge meter for electric vehicles. 


\subsection{Data processing software}

Commonly used methods for cycle construction are microtrip-based cycle construction, segmentbased cycle construction, modal cycle construction and pattern classification cycle construction.

With the microtrip method, a drive cycle is constructed by chaining representative micro-trips and matching them as closely as possible to the recorded data. The major limitation of this method is that the beginnings and ends of micro-trips are based on specific speed, acceleration, and duration constraints. The micro-trip method has been mostly used to develop drive schedules representative of a single trip. [35] [41] [43] [47]

The segment based cycle construction consists in the development of drive cycles affected by changes in roadway type or level of service, in addition to stops. The segment based method was used in the development of the default drive cycles by roadway type. [41] Although each method has inherent advantages, disadvantages and limitations, the modal cycle construction method accurately captures regional driving modes and partitions the data into snippets, even in areas with less or no idling states, such as expressways. [42]

Machine learning methods, different types of neural networks were widely used in pattern classification cycle construction, such as

- Support Vector Machine (SVM) [34] [53],

- Learning Vector Quantization (LVQ) [54],

- Recurrent Neural Network [55],

- logistic regression [49], and

- elbow method [48].

Commonly used algorithms in data processing include

- Root Means Square analysis [31] [39] [56],

- the Markov Chain [56] [57],

- Monte-Carlo method [36],

- Sliding Window method [42] [58],

- Fisher Discriminant Analysis method [59],

- the Simulated Annealing Optimization algorithm [42], and

- Principal Component Analysis (PCA) or Canonical Discriminant Analysis (CDA). [60]

The k-means clustering [34] [57], or its variation of the k-medoids algorithm [48] [57], is widely used for clustering and classifying microtrips.

The use of MATLAB software to work with more complex algorithms in data processing can be a good solution as it involves the implementation of the algorithms [36]. For simpler tasks and methods, we can also process our own software, such as Microsoft Excel VBA macro [39], JAVA-based software (DCC2014) [56], or LabView (National Instruments) based software (DIAdem v2012). [35] 


\section{Methods of data processing}

Most of the existing driving cycles are developed based on two categories of methods. [62]

In the first method, acceleration, idle speed, deceleration, and various cruising driving modes are combined to constitute a typical driving cycle, while in the second method, the driving cycle is derived from a large quantity of real-world statistical data.

The latter method has been widely adopted for driving cycle development [62] and they can be further classified into the four categories: Random-selection-based methods; Clustering methods; Statisticalanalysis-based methods; Markov chain methods.

In the relevant publications the following techniques frequently appear

- Markov chains/processes

- Cluster analysis

- Principal Component Analysis

- Discriminant analysis

- Correspondence Analysis

- Genetic algorithm

- Neural networks

- Stochastic dynamic programming

- Deterministic dynamic programming

- Quadratic programming

- Finite-horizon stochastic dynamic programming

- Filtering methodologies

- Exponential smoothing method

- Epanechnikov Kernel smoothing algorithm

- Wavelet analysis

The typical collected data are [84]

- average speed of the entire driving cycle;

- average running speed;

- average acceleration;

- average deceleration;

- average micro-trip duration;

- time proportions of driving modes for idling;

- time proportions of driving modes for acceleration;

- time proportions of driving modes for cruising;

- time proportions of driving modes for creeping; 
- time proportions of driving modes for deceleration;

- average number of acceleration-deceleration changes;

- root mean square acceleration (arms);

- positive kinetic energy.

According to [61] the fuel economy and exhaust emission factors of the vehicles are greatly dependent on drivers, weather, road and traffic conditions. Thus, the improvement of the fuel economy and exhaust emissions should be focused on eco-driving, from the points of the driver behaviours and route-choice, which were human-controllable factors. In eco-driving, drivers also played an extremely important effect on fuel economy and exhaust emissions. The eco-driving includes both the fuel economy improvement and exhaust emission decrease. Examples for eco-driving factors are drag coefficient, road grade, wind velocity, gear shift perfection, coolant temperature, rolling resistance coefficient, vehicle velocity and mass. The cold start and warm up process need to be taken into consideration. [61]

In [62] micro-trips are determined and three representative parameters are derived from the vehicular model. Then the development of the driving cycle is transferred to an optimization problem, and a two-layer optimization method is proposed to construct the typical driving cycle. In the first layer, the optimal combination of micro-trips is determined using a genetic algorithm with varying quantity of micro-trips, whereas in the second layer, the best quantity of micro-trips is determined according to the speed-acceleration probability distribution and average energy consumption [62].

[63] aims to provide an alternative perspective in the selection of assessment measures. The engine speed and torque are used as the additional parameters, on top of the conventional vehicle parameters, in constructing the driving cycle. Furthermore, a Two-Step clustering method provided by IBM's SPSS software is applied in recognising the characteristics during the actual driving. The collected data is processed by breaking the recorded trips into smaller trips known as micro-trip. The vehicle and engine parameters are simplified through principal component analysis and followed by clustering the data using Two-Step technique. The means of the generated clusters are used as the target criterion in selecting eligible micro-trips as the sub-cycles. The sub-cycles are arranged and constructed as the initial Malaysian urban drive cycle of this study. 11 common vehicle parameters and six engine parameters which is used in the study to characterise the behaviours and identifying the trend. The parameters used in the clustering analysis are important as they may affect the integrity of the final drive cycles to represent the actual driving conditions. With high number of parameters to analyse, multivariate analysis is applied to reduce the complexity in the multi-dimensional space of the selected parameters. Principal Component Analysis is one of the multivariate analysis used in the development of drive cycles as a process to identify only the relevant parameters prior to clustering. [63]

In [64] the model is estimated and validated against real-world data, collected on a fleet of five EFVs in the city centre of Rome, for a total of 144 observed trips between subsequent pickup/delivery stops. Data included latitude and longitude, vehicle powertrain data, specifically voltage and current at the battery, and the instantaneous total weight of the vehicle collected at $1 \mathrm{~Hz}$. [64] 
[65] proposes a novel statistical description of the physical properties of road transport operations by using stochastic models arranged in a hierarchical structure. The description includes speed signs, stops, speed bumps, curvature, topography, road roughness and ground type, with a road type introduced at the top of the hierarchy to group characteristics that are often connected. Paper [65] aims to answer two questions: 'how can a road be described from a statistical point-of-view?' and 'how can that description be linked to the OC format?'. The authors do this by isolating the properties of the road and, one-by-one, formulating a stochastic model that is linked to the underlying physics. The parameters and distributions describing those models do contain information on what is typical for the transport operation in question. They offer an abstract and highly compact way to describe the driving environment. In addition, the choice of stochastic models as statistical descriptors has the added advantage that it enables the synthesis of new data. A stochastic operating cycle is obtained using the Markov model, where the transition matrix is estimated from measurements of vehicle speed. [65]

In [66] road type data are considered to improve the representativeness of constructed cycle using the conventional Markov chain method for real-world driving data. 12 parameters are extracted, which describe the characteristics of driving cycle, to indicate the differences among the constructed driving cycle, NEDC and real-world driving data. The data used in the paper are derived from the Nationalwide Inspect and Management Center for Electric Vehicle. The operation data, including vehicle data, battery data, motor data and fault data, are collected and uploaded to the center by the intelligent terminal in vehicle. The driving data downloaded from the center are divided into a large number of driving fragments. Each driving fragment consists of several micro-fragments: idle, acceleration, deceleration and cruise. [66]

Data for a total of 114 bus trips are collected in [67]. After exponential smoothing, target statistics for 13 assessment parameters are derived using the whole data population. A driving cycle was then developed by randomly selecting micro-trips with assessment parameters closest to the target statistics. A representative driving cycle is generated according to the Markov Chain theory using a transition probability matrix derived from the whole data set. The best cycle is selected based on the difference between the Speed Acceleration Frequency Distributions of the candidate cycle and the whole data set. Principal component analysis and k-means clustering methods are adopted to analyse the data. [67]

By analysing the correlation between parameters, average speed mean, cruise time percentage drive and driving distance are chosen as the characteristic parameters in [68]. The k-means clustering method is used to divide driving cycles into four patterns. In order to improve the performance of the clustering algorithm, the simulated annealing algorithm is employed to optimize the initial cluster centres and generate random disturbance to clustering results. [68]

A stochastic driving cycle is constructed to more accurately model the dynamic characteristics of the uncertain driving cycles, derived from the historic record of individual drivers in [69]. Finite-horizon stochastic dynamic programming is adapted to globally optimize the vehicle performance in stochastic sense. If the driving cycle is known a priori, deterministic dynamic programming, quadratic programming and neural network methods could be used to solve this globally optimal problem. [69] 
Paper [70] presents an analysis which shows the zones of the cycle that are causing the highest emissions, using two different approaches. Furthermore, the paper demonstrates that it is important to include a cold start to ensure rapid warm up of the catalysts. The paper analyses the second-bysecond emission results of FTIR measurements to investigate which parts of the test cycles are causing the highest emissions. [70]

The methodology of a large-scale duty-cycle statistical analysis are presented, including graphical and tabular representations of a number of relationships between key duty-cycle metrics observed within the larger data set in [71]. Examining a number of metrics associated with vehicle energy consumption and driving behaviour, statistical relationships between key metrics of interest were explored. The raw drive cycles are filtered and analysed using NREL's Drive Cycle Rapid Investigation, Visualization, and Evaluation tool (DRIVE) to generate individual drive cycle summary statistics. To exploring the influence of geography on vehicle operation, the bivariate analyses are performed to study any potential relationships between variables. Examining the correlation between average driving speed and kinetic intensity, one observes a strong power correlation between the two variables. [71]

Extensive duty-cycle statistical analyses are performed to examine and characterize common vehicle dynamics trends and relationships based on in-use field data in [72]. Having received in-use data, the data are processed using a series of semi-automated routines. Kinetic intensity is a measure of the hybrid advantage as a ratio of characteristic acceleration to aerodynamic speed. NREL's DRIVE tool employs a deterministic multivariate hierarchical clustering method to generate representative drive cycles from source data [72].

According to [73] it is important to define a suitable set of assessment criteria with reasonable representativeness for cycle synthesis. Assessment criteria are necessary for three purposes: (1) extraction of driving characteristics; (2) construction of candidate driving cycles; and (3) determination of the statistical representativeness of the candidate driving cycles and the selection of the 'best' cycle. It would directly affect the quality of the resultant cycle. The methods concerning succession probabilities, including the Markov Chain and simulation approaches commonly consider fewer assessment criteria but usually use speed/acceleration distributions. Conversely, the matching approach tends to introduce more assessment criteria. These phenomena are basically in line with the principles behind the two approaches. The matching approach tries to 'match' the statistical characteristics of the driving data; thus a more comprehensive list of assessment criteria is required to ensuring the statistical representativeness. Conversely, the simulation approach 'uses' the driving behaviour characterized by the speed/acceleration distribution. [73]

On the basis of kinematics fragments, the characteristic parameters of kinematic sequence are carried out with the principal component analysis in [74]. Then driving cycle are synthesized based on dynamic clustering algorithm analysis to get the vehicle's driving state in different road conditions.

Candidate drive cycles are subsequently synthesized using Markov Chain based on Transition Probability Matrixes for each category in [75]. Then representative synthetic drive cycles are selected through assessment of significant cycle metrics to identify the ones with smallest errors. 
The purpose of paper [76] is to propose a driving condition cluster analysis methodology to establish the relationship of vehicle fuel consumption and driving condition type. The DBSCAN algorithm can separate high-density regions from a sample space and take isolated points in low-density regions as noise. Compared with the k-means algorithm, the DBSCAN algorithm is more suitable for seeking sample distribution centers of its natural existence. [76]

The kinematic segments are described by their idling duration and cross-distribution of the instantaneous speeds and accelerations (i.e. time-frequencies are analysed) in [77]. Correspondence Analysis (based on chi-squared distance) and Clustering tools are used to classify these segments according to their speed-acceleration distribution.

In [78] a stochastic and statistical methodology is used to develop and assess the representativeness of the driving cycle against a separate set of real-world electric vehicle driving data and the developed cycle performs well in that comparison. The Epanechnikov Kernel smoothing algorithm, with a bandwidth of three, is applied to the logged velocity data and the instantaneous acceleration rates are computed on the basis of the smoothed velocities. Subsequent to the application of the smoothing algorithm, the velocity and acceleration measurements of each cycle are inspected and the cycle is excluded from the analysis if the performance capabilities of the vehicle are exceeded. Fifty-five probability density functions were fitted iteratively to the individual journey distances to find the best fit. The three-parameter fatigue life distribution is found to be the best fit for the data based on the Kolmogorov Smirnov goodness of fit test. A driving condition recognition tool based on a learning vector quantisation neural network is developed to classify the driving cycle data as operating in particular driving conditions determined by road-type and level of congestion. The authors clustered the driving cycle variables 'idle time percentage' and 'average acceleration' of 150 micro segments into three clusters representing different traffic conditions. k-means clustering is used to cluster the congestion index and average velocity of the micro-segments into three clusters representing three levels of congestion, stop-start, congested and free-flowing respectively. The methodology for generating the driving cycles is based on Markov process theory. [78]

The Beijing driving cycle is developed using statistics and Markov chain method in [79]. The distribution of single-trip driving mileage and the distribution of single-trip travel time are used. For understanding the characteristics of driving cycles in depth, the paper defines some variables to characterize a driving cycle: Distribution of speed interval, Distribution of acceleration interval, Speed acceleration frequency distribution. [79]

In [80] the stochastic method divides a speed trace into modal events (e.g., cruise, idle, acceleration, or deceleration) and describes the sequencing of those events using Markov process theory. The benefits of this stochastic method are that partitioning of the modal events is an endogenous outcome of the speed profile without arbitrarily breaking a speed trace according to zero speeds or stops, and therefore maintains the integrity of a vehicle operation mode. The Markov modal events reveal the stochastic nature of the driving data. [80]

Regarding the cycle construction method, a random selection approach is adopted in [81]. The collected trip data sets are classified according to vehicle types. Mean values of a set of assessment criteria are then derived and set to be the target summary statistics for each vehicle type. To construct 
a candidate driving cycle, micro-trips bounded by idling times are first identified for each trip. They are then selected randomly to form a driving cycle and idling periods are also appended to the beginning of each micro-trip until the designated cycle duration is achieved. 10 acceptable cycles were generated for each vehicle type for the selection of the most representative driving cycle. Eventually, the acceptable cycle with the smallest average absolute percentage error would be selected as the most representative driving cycle for the corresponding group. [81]

Study [82] has developed a distance-based driving cycle. A computer program is developed to randomly select the micro-trips until it reached the target distance. The mean value of 14 driving parameters are identified. The percent number of micro-trips by road type and percent time spent in each road type are also calculated to further improve the representation of observed data. The developed driving cycle is accepted if the difference was less than 5 percent of the target value. Otherwise, it is ignored and the whole procedure is repeated to develop another driving cycle. By repeating this procedure, about five driving cycles are developed from the collected data. [82]

In [83] the data analysis is carried out in two parts as: analysis of base data and the analysis of microtrips. The analysis of base data involves the development of two matrices, namely speed-acceleration frequency matrix and normalized speed-acceleration matrix. The speed-acceleration frequency matrix is the frequency of occurrence of acceleration, deceleration, cruise and idle corresponding to a speed values. From the normalized matrix several parameters representing travel characteristics are computed. These (target) parameters include the percent time in acceleration, percent time in deceleration percent time in cruise, percent time in idle and average velocity. To further analyse the data, a computer program is used, which generates a driving cycle according to the proposed procedure. The program compares all the micro-trips with each other with respect to the calculated parameters of the individual micro-trips within a tolerance limits. [83]

Since Driving Cycles are coming from some kind of synthesis algorithm, the identification of numerical characteristics of input data permits to validate the representativeness of the compressed cycles. According to [84] at least six main categories of driving segment/cycles parameters can be distinguished depending on their physical dimension: distance, time, speed, acceleration, stop data, indicators of dynamics. Considering relevant experiences such as the ARTEMIS project, the full list of the parameters used includes 40 elements. In addition to this, the analysis of cycles can take into account a large set of data represented in the form of statistical distribution. Typical data can be calculated in terms of absolute indicators (e.g. distance driven or time spent over a certain condition) or in terms of relative frequencies (e.g. percentage of occurrence of a certain class of events). Regarding 2-variables distribution, a largely used method for cycle clustering and cycle build-up is based on the analysis of the distribution of events falling in a determined class of speed-acceleration couple, expressed as relative frequency of occurrence or as total time. In case of the use of such information for the extrapolation of a new cycle, the relative frequency assumes the meaning of "probability" of a determined class. The ability to categorize the different segments of measured driving sequences is fundamental for cycle synthesis; clustering activities can be based on a vector of indicators. Regarding GPS data, there are different suitable filtering methodologies, each one 
presenting advantages and disadvantages: piecewise polynomial regression model; kernel-based smoothing methods, Kalman filter. [84]

In [85] a vehicle dynamics model and a car-following model are used to establish the two-dimensional Markov state transition model. Generally, driving cycles are considered as a stochastic process, which include the orthogonal increment process, the independent increment process, the Markov process, the normal process and so on. [85] presents a full validation that vehicle driving cycles are Markov process. In the study, wavelet theory is applied to analysing the components of velocity and decomposing driving cycle information into several signals with different frequency. [85]

In [86] Tianjin driving cycle is developed by using linear discriminant analysis, which is a multivariate statistical method to discriminate samples. Different from cluster analysis, discriminant analysis is a statistical technique for classifying an observation into one of several a priori groups that depend upon the observation's individual characteristics, it is a very popular and reliable statistical method in classifying where the dependent variable appears in qualitative form and has been successfully applied to a variety of practical problems. Paper [86] analyses two states of traffic which are congestion and smooth, using two-class Fisher's discrimination.

In [87] a novel method is described and applied to generate a dynamic driving cycle that statistically matches the real-life operation of a vehicle. Two micro trip models with increasing complexity are described and fit to the data set. A micro trip model is mathematically fitted to each individual micro trip, keeping track of the integral root mean square (RMS) error between the model and measurements. [87]

Driving cycles are generated from real-world driving data using a Markov chain approach in [88]. Then these driving cycles are determined if they are candidate driving cycles that pass the selection criterion, and finally are transformed to equivalent driving cycles. In order to extract information from the real-world driving cycles and use it in a compact way the transition probability matrix is used. The transition probabilities represent the Markov chain and are used to generate driving cycles. [88]

It is presented in [89] that the selection of performance measures or assessment measures we use to analyse the driving dataset determines the quality of developed driving cycles since assessment measures are the Measure of Effectiveness in comparing the trend and characteristics of base data with those of cycles. The candidate driving cycle with the least values of assessment measures is usually selected as the final cycle since it can approximately replicate the trend and characteristics of the collected whole driving dataset. [89]

Since the traffic process of vehicle is random, the driving cycle of vehicle is studied by the Markov theory in [90]. By analysis and calculating a number of experimental data, the transition probability matrix of original data is obtained by maximum likelihood estimation to determine the statistical characteristics of the experimental data then according the constraints of the transfer matrix, a large number of model events were selected from experimental data randomly to develop a driving cycle. [90] 


\section{Conclusion}

The goal of this paper is to give an overview of the relevant literature with the motivation of construction our own driving cycles in the future. Many studies have shown that the general driving cycles are not accurate enough to adequately estimate emissions and energy use, therefore the need for more representative, special driving cycles is valid and justifiable. Accordingly, many different driving cycles have been published in recent years, all of which are indeed very different from the global driving cycles. The literature overview covers the papers dealing with sample route determination, data collection and processing, driving cycle construction procedures, statistical evaluation of data are in the focus of this literature research.

\section{Acknowledgement}

The research was financed by the Thematic Excellence Programme of the Ministry for Innovation and Technology in Hungary (ED_18-1-2019-0028), within the framework of the (Automotive Industry) thematic programme of the University of Debrecen.

\section{References}

[1] Fei Zhang, Fen Guo, Hong Huang: A Study of Driving Cycle for Electric Special-purpose Vehicle in Beijing , Energy Procedia 105:4884-4889

[2] J. H. Kent, G. H. Allen, G. Rule: A driving cycle for Sydney, Transportation Research, Volume 12, Issue 3, June 1978, Pages 147-152

[3] Schifter I, Díaz L, Rodríguez R, López-Salinas E.: A driving cycle for vehicle emissions estimation in the metropolitan area of Mexico City, Environ Technol. 2005 Feb;26(2):145-54.

[4] H. Y. Tong, W. T. Hung: A Framework for Developing Driving Cycles with On-Road Driving Data, Transport Reviews Volume 30, 2010 - Issue 5

[5] JieLin, Debbie A, Niemeier: An exploratory analysis comparing a stochastic driving cycle to California's regulatory cycle, Atmospheric Environment Volume 36, Issue 38, December 2002, Pages 5759-5770

[6] Michel André, Mario Rapone: Analysis and modelling of the pollutant emissions from European cars regarding the driving characteristics and test cycles, Atmospheric Environment Volume 43, Issue 5, February 2009, Pages 986-995

[7] Hung WT, Tam KM, Lee CP, Chan LY, Cheung CS.: Characterizing driving patterns for Zhuhai for traffic emissions estimation, J Air Waste Manag Assoc. 2006 Oct;56(10):1420-30.

[8] José Ignacio Huertas, Luis Felipe Quirama, Michael Daniel Giraldo, Jenny Díaz: Comparison of driving cycles obtained by the micro-trips, markov-chains and mwd-cp methods, International Journal of Sustainable Energy Planning and Management, Volumen 22, 109-120 
International Journal of Engineering and Management Sciences (IJEMS) Vol. 5. (2020). No. 2

DOI: 10.21791/IJEMS.2020.2.31.

[9] José Ignacio Huertas, Luis Felipe Quirama, Michael Giraldo, Jenny Díaz-Ramírez: Comparison of Three Methods for Constructing Real Driving Cycles, Energies 12(4):665, 2019

[10] Xuan Zhao, Jian Ma, Shu Wang, Yiming Ye, Yan Wu, Man Yu: Developing an electric vehicle urban driving cycle to study differences in energy consumption, Environmental Science and Pollution Research, Volume 26, Issue 14, pp 13839-13853 May 2019

[11] Sze-Hwee Ho, Yiik-Diew Wong, Victor Wei-Chung Chang: Developing Singapore Driving Cycle for passenger cars to estimate fuel consumption and vehicular emissions, Atmospheric Environment Volume 97, November 2014, Pages 353-362

[12] H. Y. Tong, H. D. Tung, W. T. Hung, H. V. Nguyen: Development of driving cycles for motorcycles and light-duty vehicles in Vietnam, Atmospheric Environment Volume 45, Issue 29, September 2011, Pages 5191-5199

[13] Monica Tutuianu et al.: Development of the World-wide harmonized Light duty Test Cycle (WLTC) and a possible pathway for its introduction in the European legislation, Transportation Research Part D: Transport and Environment 40:61-75, 2015

[14] P. Seers, G. Nachin, M. Glaus: Development of two driving cycles for utility vehicles, Transport and Environment Volume 41, December 2015, Pages 377-385

[15] H. Y. Tong: Development of a driving cycle for a supercapacitor electric bus route in Hong Kong, Sustainable Cities and Society Volume 48, July 2019, 101588 [15] H.Y.Tong: Development of a driving cycle for a supercapacitor electric bus route in Hong Kong, Sustainable Cities and Society, Volume 48, 2019

[16] H. Y. Tong, W. T. Hung, C. S. Cheung: Development of a driving cycle for Hong Kong, Atmospheric Environment, Volume 33, Issue 15, 1 July 1999, Pages 2323-2335

[17] W. T. Hung, H. Y. Tong, C. P. Lee, K. Ha, L. Y. Pao: Development of a practical driving cycle construction methodology: A case study in Hong Kong, Transportation Research Part D: Transport and Environment, Volume 12, Issue 2, March 2007, Pages 115-128

[18] Peng Yuhui, Zhuang Yuan, Yang Huibao: Development of a representative driving cycle for urban buses based on the K-means cluster method, Cluster Computing, May 2019, Volume 22, Supplement 3, pp 6871-6880

[19] Shen Peihong, Zhao Zhiguo, Li Jingwei, Zhan Xiaowen : Development of a typical driving cycle for an intra-city hybrid electric bus with a fixed route, Transportation Research Part D: Transport and Environment, Volume: 59, pp 346-360

[20] A., Geetha, Subramani, C.: Development of driving cycle under real world traffic conditions: A case study, International Journal of Electrical and Computer Engineering (IJECE), V 9, pp47984803, 2019

[21] J. B. Manuel M. Biona, Alvin B. Culaba: Drive cycle development for tricycles, Clean Technologies and Environmental Policy, May 2006, Volume 8, Issue 2, pp 131-137 
International Journal of Engineering and Management Sciences (IJEMS) Vol. 5. (2020). No. 2

DOI: 10.21791/IJEMS.2020.2.31.

[22] Wenyu Zhou, Ke Xu, Ying Yang, Jiahuan Lu: Driving Cycle Development for Electric Vehicle Application using Principal Component Analysis and K-means Cluster: With the Case of Shenyang, China, Energy Procedia, Volume 105, May 2017, Pages 2831-2836

[23] Jin Zhang, Zhenpo Wang, Peng Liu, Zhaosheng Zhang, Xiaoyu Li, Changhui Qu: Driving cycles construction for electric vehicles considering road environment: A case study in Beijing, Applied Energy, Volume 253, 1 November 2019,

[24] Peter Nyberg, Erik Frisk, Lars Nielsen: Generation of Equivalent Driving Cycles Using Markov Chains and Mean Tractive Force Components, IFAC Proceedings Volumes Volume 47, Issue 3, 2014, Pages 8787-8792

[25] Li Yuecheng, Peng Jiankun, Hongwen he, Xie Shanshan: The Study on Multi-scale Prediction of Future Driving Cycle Based on Markov Chain. Energy Procedia. 105. 3219-3224. 2017

[26] T. J. Lyons, J. R. Kenworthy, P. I. Austin, P. W. G. Newman: The development of a driving cycle for fuel consumption and emissions evaluation, Transportation Research Part A: General, Volume 20, Issue 6, November 1986, Pages 447-462

[27] Esteves-Booth A., Muneer T., Kirby H., Kubie J., Hunter J.: The measurement of vehicular driving cycle within the city of Edinburgh. Transportation Research Part D-transport and Environment 6. 209-220

[28] Eva Ericsson: Variability in urban driving patterns, Transportation Research Part D: Transport and Environment, Volume 5, Issue 5, 2000, Pages 337-354

[29] Daniel Chindamo, MarcoGadola: What is the Most Representative Standard Driving Cycle to Estimate Diesel Emissions of a Light Commercial Vehicle? IFAC-PapersOnLine, Volume 51, Issue 5, 2018, Pages 73-78

[30] Donateo Teresa, Giovinazzi Mattia: Building a cycle for Real Driving Emissions. Energy Procedia. 126. 891-898. 2017.

[31] N.H. Arun, Srinath Mahesh, Gitakrishnan Ramadurai, S.M. Shiva Nagendra - Development of driving cycles for passenger cars and motorcycles in Chennai, India - Sustainable Cities and Society 32 (2017) 508-512

[32] Ross Milligan, Saioa Etxebarria, Tariq Muneer and Eulalia Jadraque Gago - Driven Performance of Electric Vehicles in Edinburgh and Its Environs - Energies (2019), 12, 3074

[33] Wafaa Saleh, Ravindra Kumar, Anil Sharma - Driving cycle for motorcycles in modern cities: case studies of Edinburgh and Delhi - World Journal of Science, Technology \& Sustainable Development,(2010) Vol. 7, No. 3

[34] Xuan Zhao, Qiang Yu, Jian Ma, Yan Wu, Man Yu, and Yiming Ye - Development of a Representative EV Urban Driving Cycle Based on a k-Means and SVM Hybrid Clustering Algorithm - Journal of Advanced Transportation (2018) Article ID 1890753 
International Journal of Engineering and Management Sciences (IJEMS) Vol. 5. (2020). No. 2

DOI: 10.21791/IJEMS.2020.2.31.

[35] Habib Kaymaz, Hayriye Korkmaz, Hasan Erdal - Development of a driving cycle for Istanbul bus rapid transit based on real-world data using stratified sampling method - Transportation Research Part D 75 (2019) 123-135

[36] Klaus Kivekäs, Antti Lajunen, Jari Vepsäläinen and Kari Tammi - City Bus Powertrain Comparison: Driving Cycle Variation and Passenger Load Sensitivity Analysis - Energies (2018), 11,1755

[37] José I. Huertas, Michael Giraldo, Luis F. Quirama and Jenny Díaz - Driving Cycles Based on Fuel Consumption - Energies (2018), 11, 3064

[38] Marguerite Nyhan, Stanislav Sobolevsky, Chaogui Kang, Prudence Robinson, Andrea Corti, Michael Szell, David Streets, Zifeng Lu, Rex Britter, Steven R.H. Barrett, Carlo Ratti - Predicting vehicular emissions in high spatial resolution using pervasively measured transportation data and microscopic emissions model - Atmospheric Environment 140 (2016) 352 - 363

[39] Jean-Baptiste Gallo - Development of Representative Regional Delivery Drive Cycles for HeavyDuty Truck Tractors - SAE International Journal of Commercial Vehicles (2014) Volume7, Issue1

[40] Pantitcha Outapa, Sarawut Thepanondh, Akira Kondo, and Natchanok Pala-En - Development of air pollutant emission factors under real-world truck driving cycle - International Journal of Sustainable Transportation (2018), Vol. 12, No. 6, 432-440

[41] Junshi $\mathrm{Xu}$, Marc Saleh, An Wang, Ran Tu, Marianne Hatzopoulou - Embedding local driving behaviour in regional emission models to increase the robustness of on-road emission inventories - Transportation Research Part D 73 (2019) 1-14

[42] Renjuan Sun, Yuxin Tian, Hongbo Zhang, Rui Yue, Bin Lv, and Jingrong Chen - Data-Driven Synthetic Optimization Method for Driving Cycle Development - IEEE Access (2019) 10.1109/ACCESS.2019.2950169

[43] P. Lipar, I. Strnad, M. Česnik, T. Maher - Development of Urban Driving Cycle with GPS Data Post Processing - Promet Traffic \& Transportation, (2016) Vol. 28, No. 4, 353-364

[44] Xiaoyi He, Ye Wu, Shaojun Zhang, Michael A. Tamor, Timothy J. Wallington, Wei Shen, Weijian Han, Lixin Fu, Jiming Hao - Individual trip chain distributions for passenger cars: Implications for market acceptance of battery electric vehicles and energy consumption by plug-in hybrid electric vehicles - Applied Energy 180 (2016) 650-660

[45] Adam Duran and Matthew Earleywine - GPS Data Filtration Method for Drive Cycle Analysis Applications - SAE International (2012) NREL/CP-5400-53865.

[46] Li Shen and Peter R. Stopher - Review of GPS Travel Survey and GPS Data-Processing Methods Transport Reviews (2014) Vol. 34, No. 3, 316-334

[47] Carlos A. Romero, Luz A. Mejía, Ricardo Acosta - Engine data collection and development of a pilot driving cycle for Pereira city by using low cost diagnostic tools - Ingeniería y Competitividad (2017) Volumen 19, No. 2, p. 11 - 24 
[48] Robert Prohaska, Arnaud Konan, Kenneth Kelly, and Michael Lammert - Heavy-Duty Vehicle Port Drayage Drive Cycle Characterization and Development - SAE International Journal of Commercial Vehicles (2016) Volume 9, Issue 2

[49] Lei Zhu, Jeffrey D. Gonder - A driving cycle detection approach using map service API Transportation Research Part C 92 (2018) 349-363

[50] Runze Hao, Shupeng Zhao, Ning Li, Lei Liang - Vehicle Driving Data Collection and Analysis Based on GPRS - Sensors \& Transducers (2014), Vol. 166, Issue 3, pp. 23-28

[51] Jing Lei, Wan Ren Jun, Wei Ke Xin - Design of Driving Cycle Data Acquisition System Based on GPS - Applied Mechanics and Materials (2013) Vol. 391 pp 572-575

[52] A. Loiselle-Lapointe, A. J. Conde and H. Ribberink - Effects of Drive Cycle, Ambient Temperature and Accessory Usage on Energy Consumption and All-Electric Range - International Journal of Automotive Technology (2017), Vol. 18, No. 1, pp. 103-115

[53] Qin Shi, Duoyang Qiu , Lin He, BingWu and Yiming Li - Support vector machine-based driving cycle recognition for dynamic equivalent fuel consumption minimization strategy with hybrid electric vehicle - Advances in Mechanical Engineering (2018), Vol. 10(11) 1-17

[54] Gengchen Liu, Jianwu Zhang - An energy management of plug-in hybrid electric vehicles based on driver behavior and road information - Journal of Intelligent \& Fuzzy Systems 33 (2017) 30093020

[55] Duoguan Qiu, Yuan Li, Dapeng Qiao - Recurrent Neural Network Based Driving Cycle Development for Light Duty Vehicles in Beijing - Transportation Research Procedia 34 (2018) 147-154

[56] Uditha Galgamuwa, Loshaka Perera and Saman Bandara - Development of a driving cycle for Colombo, Sri Lanka: an economical approach for developing countries - Journal of Advanced Transportation (2016), 50: 1520-1530

[57] Ruoyun Ma, Xiaoyi He, Yali Zheng, Boya Zhou, Sheng Lu, Ye Wu - Real-world driving cycles and energy consumption informed by large-sized vehicle trajectory data - Journal of Cleaner Production 223 (2019) 564-574

[58] Ladan Mozaffari, Ahmad Mozaffari, Nasser L. Azad - Vehicle speed prediction via a slidingwindow time series analysis and an evolutionary least learning machine: A case study on San Francisco urban roads - Engineering Science and Technology, International Journal (2014) 1-13

[59] Zhang Shupei, Zhang Wei - Research on the method of screening the real vehicle data fragment for Driving Cycle - Applied Mechanics and Materials Vols. 738-739 (2015) pp 564-568

[60] Giovanni Meccariello, Livia Della Ragione - Statistical evaluation of driving cycle with slope variability - Electronic Journal of Applied Statistical Analysis, Vol. 09, Issue 04, (2016), 610-622

[61] Jianbing Gaoa, Haibo Chena, Ying Lib, Junyan Chena, Yuanjian Zhangc, Kaushali Davea, Yue Huanga, Fuel consumption and exhaust emissions of diesel vehicles in worldwide harmonized 
International Journal of Engineering and Management Sciences (IJEMS) Vol. 5. (2020). No. 2

DOI: 10.21791/IJEMS.2020.2.31.

light vehicles test cycles and their sensitivities to eco-driving factors, Energy Conversion and Management 196 (2019) 605-613

[62] Zeyu Chen, Qing Zhang, Jiahuan Lu, Jiangman Bi, Optimization-based method to develop practical driving cycle for application in electric vehicle power management: A case study in Shenyang, China, Energy 186 (2019) 115766

[63] Mohd Azman Abasa, Srithar Rajooa, Shaiful Fadzil Zainal Abidinb, Development of Malaysian urban drive cycle using vehicle and engine parameters, Transportation Research Part D 63 (2018) 388-403

[64] Chiara Fiori, Vittorio Marzano, Modelling energy consumption of electric freight vehicles in urban pickup/delivery operations: analysis and estimation on a real-world dataset, Transportation Research Part D 65 (2018) 658-673

[65] Pär Petterssona, Pär Johannessonb, Bengt Jacobsona, Fredrik Bruzeliusa,c, Lars Fastd, Sixten Berglunda, A statistical operating cycle description for prediction of road vehicles' energy consumption, Transportation Research Part D 73 (2019) 205-229

[66] Wang, Z., Zhang, J., Liu, P., Qu, C., Li, X., Driving Cycle Construction for Electric Vahicles Based on Markov Chain and Monte Carlo Method: A Case Study in Benjing, Energy Procedia 158 (2019) 2494-2499

[67] H.Y. Tong, Development of a driving cycle for a supercapacitor electric bus route in Hong Kong, Sustainable Cities and Society 48 (2019) 101588

[68] Zhenzhen Lei, Dongye Sun, Yonggang Liu, Jie Li, Pan Zhao, Simulation and Analysis of Energy Consumption for Plug-in Hybrid Electric Vehicles Based on Driving Cycles, IFAC PapersOnLine 51-31 (2018) 394-399

[69] Hongtao Zhang, Yanzhou Qina, Xianguo Lia, Xinzhi Liub, Jinyue Yanc, Power Management Optimization in Plug-In Hybrid Electric Vehicles Subject to Uncertain Driving Cycles, eTransportation https://doi.org/10.1016/j.etran.2019.100029

[70] Joachim Demuynck, Dirk Bosteelsb, Michel De Paepea, Cécile Favreb, John Mayb, Sebastian Verhelsta, Recommendations for the new WLTP cycle based on an analysis of vehicle emission measurements on NEDC and CADC, Energy Policy 49 (2012) pp234-242, 2012

[71] Duran, A. and Walkowicz, K., A Statistical Characterization of School Bus Drive Cycles Collected via Onboard Logging Systems, SAE Int. J. Commer. Veh. 6(2):2013,

[72] Robert Prohaska, Adam Duran, Adam Ragatz, Kenneth Kelly, Statistical Characterization of Medium-Duty Electric Vehicle Drive Cycles, Conference Paper NREL/CP-5400-63607 May 2015

[73] Tong, H.Y., Hung, W.T., 2010. A framework for developing driving cycles with on-road driving data. Transportation Review 30, 589-615.

[74] Liu Y, Li J, Shen B. Research on driving cycle of long-distance passenger vehicles based on principal component analysis and cluster Algorithm. International Journal of Control \& Automation 2014, Vol 7, 125-136. 
International Journal of Engineering and Management Sciences (IJEMS) Vol. 5. (2020). No. 2 DOI: 10.21791/IJEMS.2020.2.31.

[75] Liu Z, Ivanco A, Filipi Z. Naturalistic drive cycle synthesis for pickup trucks. Journal of Safety Research, 2015, Vol 54, 109-115.

[76] Xie H, Tian G, Chen H, Wang J, Huang Y. A distribution density-based methodology for driving data cluster analysis: a case study for an extended-range electric city bus. Pattern Recognition 2017, Vol 73, 131-143.

[77] Michel Andre, The ARTEMIS European driving cycles for measuring car pollutant emissions, Science of the Total Environment, 2004, 73-84

[78] Brady J, Mahony M. Development of a driving cycle to evaluate the energy economy of electric vehicles in urban areas. Applied Energy 2016, Vol 177, 165-178.

[79] Gong H, Zou Y, Yang Q, et al. Generation of a driving cycle for battery electric vehicles: A case study of Beijing[J]. Energy, 2018, Vol 150, 901-912

[80] Fotouhi, A., Montazeri-Gh, M., 2013. Tehran driving cycle development using the k-means clustering method. Scientia Iranica, 2013, 20(2), 286-293.

[81] Tong H, Tung H, Hung W, Nguyen H. Development of driving cycles for motorcycles and lightduty vehicles in Vietnam. Atmospheric Environment, 2011, Vol 45, 5191-5199

[82] Nesamania, K. S., \& Subramanian, K. P. (2011). Development of a driving cycle for intracity buses in Chennai, India. Atmospheric Environment, 2011, Vol 45, 5469-5476

[83] Kamble S, Mathew T, Sharma G. Development of real-world driving cycle: case study of Pune, India. Transportation Research D, 2009, Vol 14, 132-140

[84] Berzi L, Delogu M, Pierini M. Development of driving cycles for electric vehicles in the context of the city of Florence. Transportation Research D, 2016, Vol 47, 299-322

[85] Shi S, Lin N, Zhang Y, Cheng J, Huang C. Research on Markov property analysis of driving cycles and its application. Transportation Research D, 2016, Vol 47, 171-181

[86] Jing Z, Wang G, Zhang S, Qiu C. Building Tianjin driving cycle based on linear discriminant analysis. Transportation Research D, 2017, Vol 53, 78-87

[87] Günther R, Wenzel T, Wegner M. Big data driven dynamic driving cycle development for busses in urban public transportation. Transportation Research D, 2017, Vol 51, 276-289

[88] Nyberg P, Frisk E, Nielsen L. Generation of equivalent driving cycles using Markov chains and mean tractive force components[J]. IFAC Proceedings Volumes, 2014, 47(3), 8787-8792

[89] Xiao, Z., Dui-Jia, Z., Jun-Min, S., 2012. A synthesis of methodologies and practices for developing driving cycles. Energy Procedia 16, 1868-1873

[90] Shi, Q., Zheng, Y., Wang, R., Li, Y., 2011. The study of a new method of driving cycles construction. Procedia Engineering 16, 2011, 79-87 